\title{
Hybrid Sensorless Motor Control in aerospace applications, a survey in sensorless control, optimizing for availability and acoustic noise
}

\author{
Stefanos Skoulaxinos \\ ECMS, \\ Collins Aerospace, \\ Shirley, Solihull, UK \\ Stefanos.Skoulaxinos@collins.com \\ \& \\ Power Electronics, Machines and \\ Control Group \\ University of Nottingham, \\ Nottingham, UK \\ Stefanos. \\ Skoulaxinos@nottingham.ac.uk
}

\author{
Pat Wheeler \\ Power Electronics, Machines and \\ Control Group \\ University of Nottingham \\ Nottingham, UK \\ Pat.Wheeler@nottingham.ac.uk
}

\author{
Gaurang Vakil \\ Power Electronics, Machines and \\ Control Group \\ University of Nottingham \\ Nottingham, UK \\ Gaurang.Vakil@nottingham.ac.uk
}

\begin{abstract}
A wide range of aerospace applications previously based on hydraulics including the control of aerodynamic surfaces are being actively replaced by lighter, more intelligent Motor Drive Electronics (MDEs). However, motor resolvers commonly used to provide the rotor's position to the MDEs are sensitive devices susceptible to extreme temperature and vibrations, conditions commonly encountered in aerospace applications. This weakness can lead to resolver failures and reduction in system availability. In DALA safety critical motor control designs, system a vailability is paramount. Following a survey of sensorless methods, this paper proposes a hybrid motor control solution where sensorless rotor position calculation is run in parallel with the resolver interface, monitoring the resolver. In the event of a resolver failure, the sensorless motor control takes over controlling the motor. Furthe rmore in order to suppress acoustic noise produ ced from High Frequency injection, a variant of the Pseudo Random High Frequency injection method is also proposed.
\end{abstract}

Keywords- permanent magnet synchronous motors, sensorless motor control, aerospace, MDEs, acoustic noise suppression

\section{INTRODUCTION}

A number of safety critical functions onboard an aircraft such as the control of aerodynamic surfaces have been commonly based in the past decades on hydraulic systems. However, hydraulics tend to be heavy, occupying large volume on an aircraft and lacking flexibility in terms of their operation, fault detection and fault reporting. There is a tendency to reduce the weight and size of components aiming to increase efficiency. It is not surprising therefore that hydraulic systems tend to be replaced by more intelligent digitally controlled Motor Drive Electronics (MDEs), capable of providing a more fuel efficient, compact, lighter and more flexible implementation.

The most commonly encountered electric motor type in MDEs for safety critical aerospace applications is the Permanent Magnet Synchronous Motor(PMSM). In order to be able to reliably control the torque and rotation of a PMSM it is vital that the controller is continuously informed of the rotor's position. This is typically performed with the help of a resolver embedded in the motor. Resolvers however can suffer fromfailure modes detailed in section III of this paper.
In the eventuality of a resolver failure the MDE is unable to drive the motor efficiently and the system becomes unavailable. Aiming to increase availability, a scheme is proposed where sensorless position calculation is run in parallel with the main resolver interface and if a resolver failure takes place sensorless motor control takes over.

This paper begins by providing a background analysis of available motor types, their merits and suitability for aerospace applications. Resolver failure modes are then pres ented illustrating the weakness of current res olver based MDEs and the need for an additional motor control method for increased robustness and availability. The paper continues by providing a survey of sensorless control methods and finally presents the proposed hybrid resolver-sensorless motor control methodology aiming to increase system availability and reduce acoustic acoustic noise.

\section{ElECTRIC MOTORS AND SUIT ABILITY FOR AEROSPACE}

A wide variety of motor types is currently available with each typecharacterized by the advancements in analog, digital and material technology. Direct Current (DC) motors establish torque and rotation with the application of a DC voltage supply. IndustrialDC motors are generally expensive and complex to manufacture, they demand high levels of maintenance, tend to be larger, heavier, than equiv alent power ACmachines and can create sparks while spinning. All of the above characteristics make DC motors a rarely encountered machine in the safety critical aero space s ector.

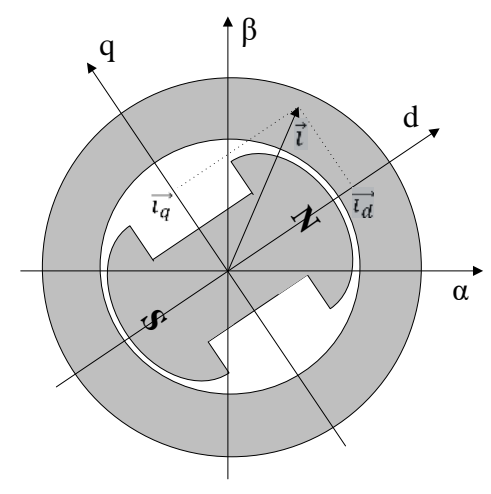

Fig. 1. PMSM stationary and rotating reference frame (RRF) currents 
Alternating Current (AC) motors can be classified into asynchronous machines (induction motors) and synchronous machines (PMSMs, Brushless DC Motors). Both types of AC machines are economical and simple to manufacture and are practically maintenance free. They are more lightweight and efficient than equivalent power DC motors. Induction motors however require excitation currents to flow through the rotor windings thus need to be of bigger physical size to prevent overheating. Brushless DC motors are considered to produce higher torque ripple than PMSMs. In conclu sion, considering the high power density of PMSMs, their dynamic performance, the relatively low manufacturing cost and lack of maintenance needs, they are considered an excellent option for high performance safety critical motor control applications in the aerospace sector. PMSMs have been commonly used for the control of aerodynamic surfaces onboard an aircraft and are the focus of this research work. Both Surface mounted Permanent Magnet Synchronous Motors (SPMSM) and Interior Permanent Magnet Synchronous Motors (IPMSM) are inves tigated under this res earch. As SPMSMs are more widely used in aerospace, results from this motor type only are pres ented in this paper.

\section{RESOLVER FAILURE MODES}

Motor resolvers are electro-mechanical components that monitor rotation and provide electrical signals conveying the position of the rotor. Faults in resolvers can be classified into the below groups:

1. A short or open takes place in the excitation transformer or in one of the stator sine and cosine windings.

\section{A short or open takes place in the resolver harness.}

3. The res olver rotor slips or becomes detached fromthe main rotor.

The first two failure modes can be detected by monitoring the res olver feedback signals. However such monitoring does not suffice to detect the third type of failure. Also upon any of the above failures a resolver based MDE would become inoperational. To detect any resolver failure mode and also allow the MDEto remain operational a sensorlessmethod can be used.

\section{SURVEY OF SENSORLESS METHODS FOR PMSMS}

In view of the disadvantages encountered in resolver based motor control designs, sensorless motor control has been an area of research in the past decades. Sensorless position calculation involves calculating the angle of a motor without the use of an encoder but by means of monitoring feedback signals such as motor voltages and currents.

Sensorless motor control algorithms for PMSMs can be classified into two main groups, model based methods and saliency based methods. The former group involves utilizing motor feedback signals during rotation to establish the rotor angle considering the equivalent circuit model of the motor. The latter group takes advantage of anisotropies in PMSMs by means of injecting High Frequency carriers and analyzing their effect to the motor's feedback signals. Model based methods are considered suitable for medium to high speed motor rotation as they rely on feedback signals while the motor is spinning while saliency methods are typically applied at standstill and low speed. Below is a survey of sensorless motor control methods for PMSMs (s ee Table 1):

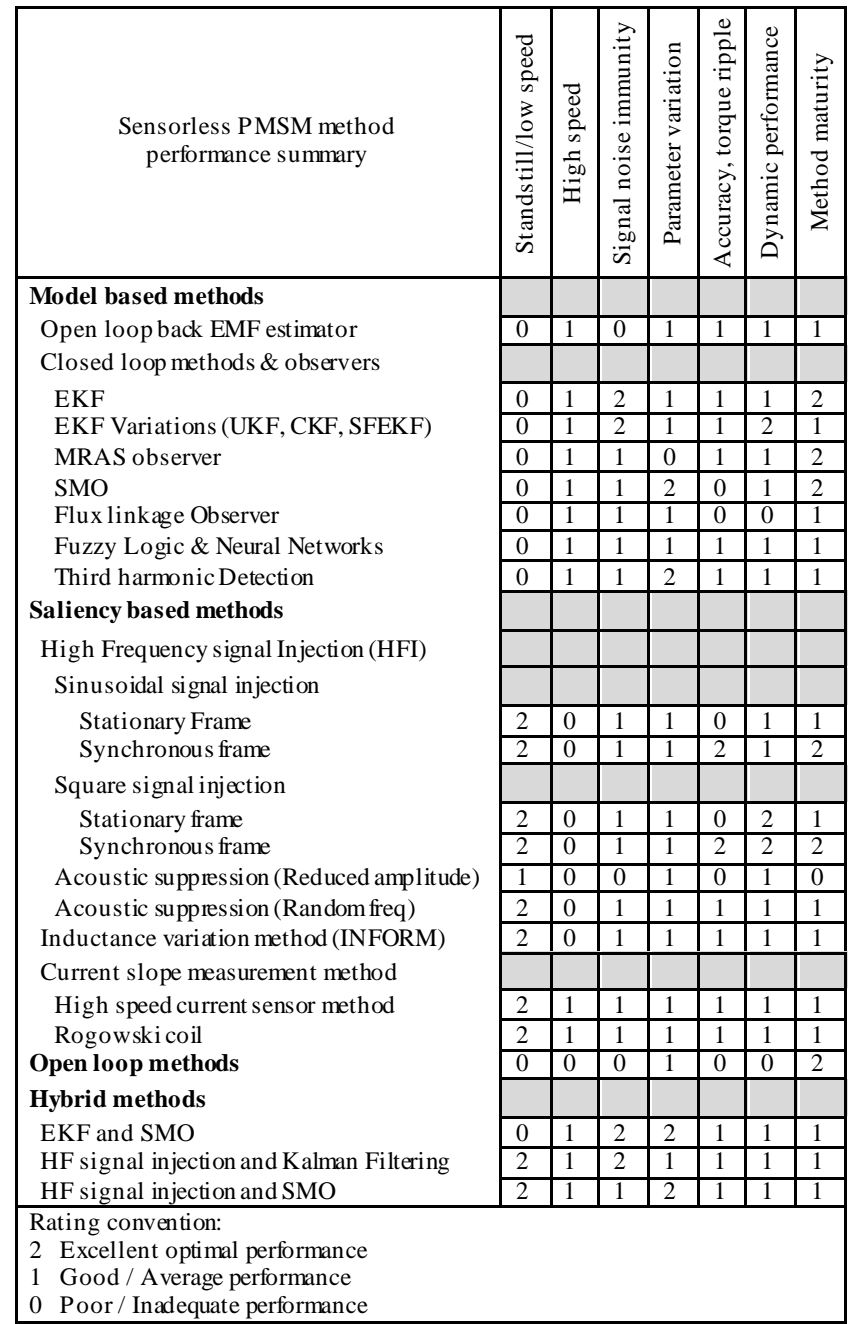

TABLE I. SENSORLESS MOTOR CONTROL METHODS

Open Loop Back Electromotive Force (EMF) estimator): The method calculates the rotor angle by in tegrating the backEMF of a spinning motor. It is simple to implement howeverbeing open loop the method leads to accumulative errors [3] due to sampling noise and DC offset while it is also vulnerable to motor parameter variation[3]. Considering the above, this method is not widely used.

Closed loop and observer methods: In order to mitigate ag ains t noise and en able self-corrective capability closed loop methods and observers were introduced:

Extended Kalman Filtering observer (EKF) and variants: The EKF is a widely used observer [1]. The method is highly effective in processing a noisy source contaminated with random interference. Limitations include the difficulty to track fast changes in motor speed and tuning complexity. To remedy the former the Sub-optimal Fading Extend Kalman Filter (SFEKF) variant was proposed [7]. To remedy the latter, the Unscented Kalman Filter (UKF) [9] and Cubature Kalman Filter (CKF) [8] were proposed. The EKF observer is considered more complex to implement than the MRAS and SMO methods.

MRAS Observer: The Model Reference Adaptive System (MRAS) observer is a widely used method relying on the application of the motor equivalent circuit to two parallel models, the reference model and the adaptive model thus the name. The accuracy of this observer is susceptible to motor 
parameter variation. Variants of the method to mitigate this limitation have been proposed in [24] where the method is less parameter sensitive.

Sliding Mode Observer (SMO): The Sliding Mode Observer (SMO) is a method designed to extract the back EMF voltage of a spinning motor and from this calculate the motor speed and angle [10]. The SMO method is simpler to implement than the EKF and is not impacted by motor parameter variation as is the MRAS. However the method is known to introduce jittery and noisy position estimation and motor control. Variants of the method have been proposed to mitigate the jittery performance including hybrid methods marrying the best characteristics of different methods such as the EKF/SMO.

Flux linkage Observer: The method calculates the rotor position by means of estimating the rotor flux [21]. The observer has a number of limitations most importantly the difficulty to accurately estimate the rotor flux. Variants of the method attempting to remedy this have been proposed including the Second Order Integral Flux Observer (SOIFO) [22]. This method however is not as widely used as the EKF, MRAS and SMO.

Fuzzy Logic and Neural Networks: The application of Neural Networks (NN), Fuzzy Logic (FL), and Fuzzy Neural Network (FNN) in the area of sens orless motor control has also been an area of active research [11]. A number of publications propose algorithms based on the above artificial intelligence methodologies towards the calculation of motor speed and position. Such methods are considered suitable for cases when the systemis difficult to model accurately due to its complexity or non-linearity for example when attempting to compen sate parameter variation.

Third harmonic detection: This method is a variant of the flux linkage calculator. Specifically the third harmonic flux linkage is estimated by means of third harmonic back EMF integration. This method was introduced by Moreira and Lipo in [23]. The method enjoys a number of advantages over a number of conventional model based sensorless methods including not being affected by parameter variation, and PWM noise [13].

High Frequency $(H F)$ signal injection: To enable sensorless motor control at zero or low speed, High Frequency signal injection was introduced. The method involves superimposing a high frequency voltage carrier to the fundamental frequency of the motor applied either at the stationary or synchronous frames and then observing the resulting motor current feedbacks. If the motor is prominently salient i.e. $\mathrm{Ld}<\mathrm{Lq}$ as is the case for an IPMSM or it becomes salient due to stator iron saturation by injecting in the direct axis of a SPMSM, the feedback currents will convey information on the position of the rotor.

Sinewave injection (rotating/pulsating): The sinusoidal rotating signal injection method involves adding a carrier signal to the alpha and beta stationary frame. Dis advantages of the method involve lack of dynamic performance due to delays associated with LPFDigital Signal Processing needed to demodulate the angle and torque ripple. Aiming to reduce the torque ripple of rotating sinusoidal method, the pulsating sinewave injection in the $\mathrm{d}$ s ynchronous axis was proposed.

Square wave injection (rotating / pulsating): The inherent delay of sinusoidal injection involving the needed low pass filters to the feedback currents, can result into low dynamic performance. To mitigate this, square signal injection was introduced. This permits higher injection frequency and more dynamic performance. Similarly it can be applied to the stationary frame and for improved torque ripple in the direct axis synchronous frame in form of pulsating square wave injection.

Acoustic noise reduction in HF injection: In order to reduce the acoustic noise caused by HF injection, one solution proposed was decreasing the amplitude of the injected signal [6]. However this also reduces the SNR and accuracy of the position. Another solution which is investigated in this research is injecting pseudorandomfrequency signal.

Inductance variation methods (INFORM): This method takes advantage of the changing magnetic conductivities in the $\mathrm{d}$ and $\mathrm{q}$ s ynchronous axis as the motor rotates [12]. It involves injecting a high frequency test voltage resulting into the transient inductance dominating over the voltage drop from the stator resistance. A variant of the method is named Indirect Flux detection by On-line Reactance Measurements (INFORM).

Current slope measurement method (high speed sensor): The current slope measurement method involves utilizing the saliency of a PMSM and detecting the rotor position by meas uring the slope of the motor currents. To do so high speed current sensors are needed and precise sampling of the current waveform to be performed in synchronism with the PWM switching.

Current slope measurement method (Rogowski coil method): Utilizing a high speed current s ensor and sampling to establish the current derivative (2.2.1) is a process where sampling needs to be carefully timed. A better method is in fact to utilise specialized hardware that measures the current derivative named Rogowski coils [15] and thus decode the rotorangle.

Open loop methods: The method is sometimes used for startup sensorless conditions when the position of the rotor can remain unknown until the motor starts spinning at which point a model based method can be used. It involves applying a sinusoidal voltage and expect the motor to rotate at this frequency. The method although simple to implement is very sensitive to load disturbance variations and provides little visibility of the motor's s tatus.

Hybrid methods: A number of hybrid methods have been introduced typically to support full speed range in sensorless mode [4] or to join the best characteristics of different sensorless methods [5].

\section{PROPOSED SENSORLESS METHOD}

The below sensorless motor control method is proposed, optimized for increased availability, reduced acoustic noise and supporting the below novel features:

1. Increased availability: the sensorless position calculation is run in parallel with the resolver interface monitoring its healthiness. If a resolver failure takes place, motor control switches to sensorless.

2. Acoustic noise reduction: Ps eudo RandomHigh Frequency injection is used to suppress acoustic noise from HFI. The reduction in acoustic noise is essential for pas sen ger comfort 
in commercial aviation and safety critical for defence aerospace applications.

3. Full speed range and dissimilar technologies: The sensorless block is to support full speed range adopting a saliency / model based hybrid method. Both FPGA (HDL) and $\mathrm{DSP}(\mathrm{C} / \mathrm{C}++)$ implementation routes are evaluated.

4. Matlab modelling and simu lation: Motor control models are maintained and simulations take place in both Matlab and implementation level simulation (HDL for FPGAs / C++ for DSP). This cros s tool comparison (Matlab/Implementation) increases simulation results confidence. The comparison of results fromindependent tools is a practice encouraged in aeros pace (DO254 standard guidance pg 78 [20]).

Related work: With regards to novelty point 1., [2] proposes a model based sensorless method to be used to monitor the resolver interface. However it provides no failure detection and no increase in availability if the failure takes place at low speed or at standstill. In contrast the research currently presented can increase availability at any speed while supporting additional features via its novelty points.

With regards to novelty point 2 there have been a number of research efforts [14], [18] primarily targeting IPMSMs. The method proposed here proposes an enhanced variant of the ps eudo randomhigh frequency injection for both IPMSMs and SPMSMs. The additional novelty features of this research als o intend to provide a more complete design methodology.

\section{A. High Frequency Injection and acoustic noise reduction (low speed):}

$\mathrm{HF}$ injection for sen sorless position calculation can introduce acoustic nois e audible to the human ear. This is due to the fact that the injected voltages produce additional torque pulsations that in turn generate acoustic noise. To suppress the acoustic noise:

1. The injection frequency is to vary in a Pseudo Random fashion. Research has shown that the human ear is more susceptible to pure tones of a specified audible frequency rather than random noise of equivalent amplitude [17].

2. Elevate the HF injection frequency and PWM frequency: Increasing the PWM switching frequency reduces the noise generated from a motor [17] as it results into s maller torque ripple. Research has also shown that the upper threshold of the human audible range is lowered with age and exposure to loud nois es down to $12 \mathrm{kHz}$ for a middle aged person [16].

The block diagram of the Pseudo RandomHigh Frequency Injection (PRHFI) proposed in this paper is illustrated in figure 2 within the saliency sensorless method subblock. The block generates pseudo random high frequency injection to identify the position of the rotor at slow speed while minimizing acoustic noise. When the motor speed is abovea threshold over which the back EMF is enough to provide accurate results, a s witch to a closed loop backEMF method takes place. Details of the implementation are als o shown in section VI of this paper.

\section{B. Closed loop Back EMF obsrver (high speed):}

The High Frequency injection is used in conjunction with a variant of a closed loop back EMF observer. The motor controller calculates the alpha and beta axis voltage demands so the voltage being injected to the motor at any point time is of known value. Themotor's R/L parameters are als o known

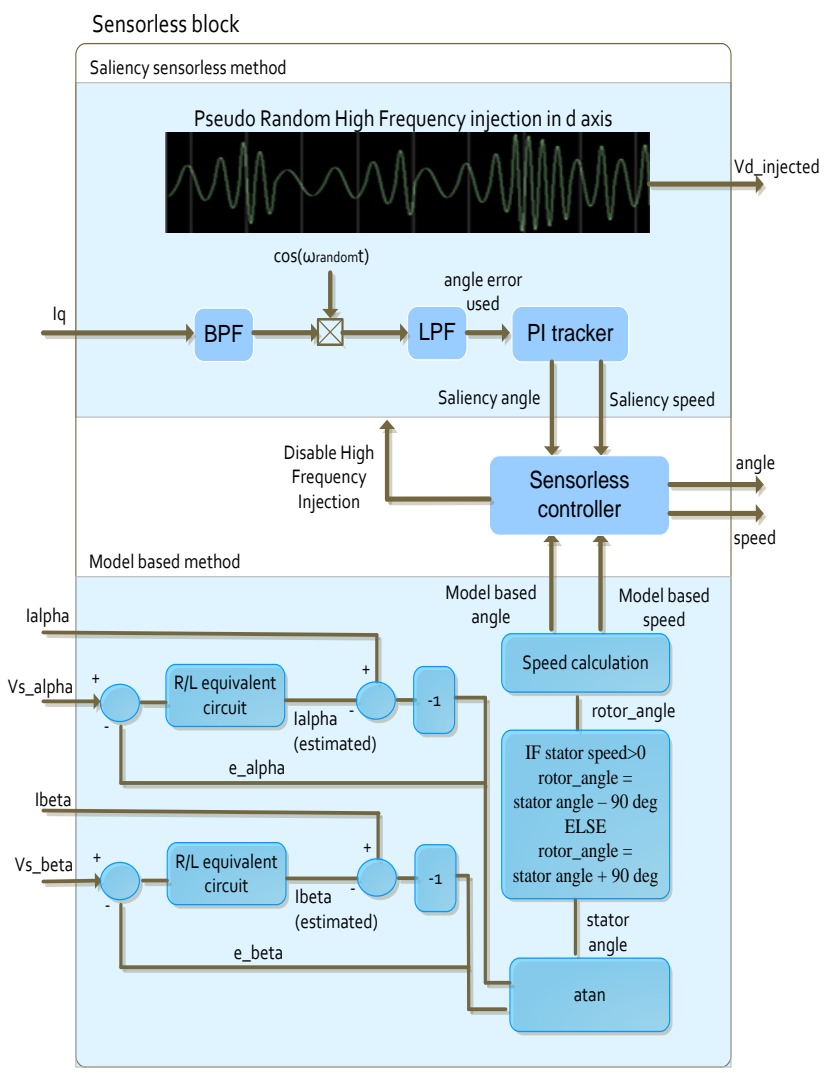

Fig. 2. Sensorless block, saliency and model based methods combined

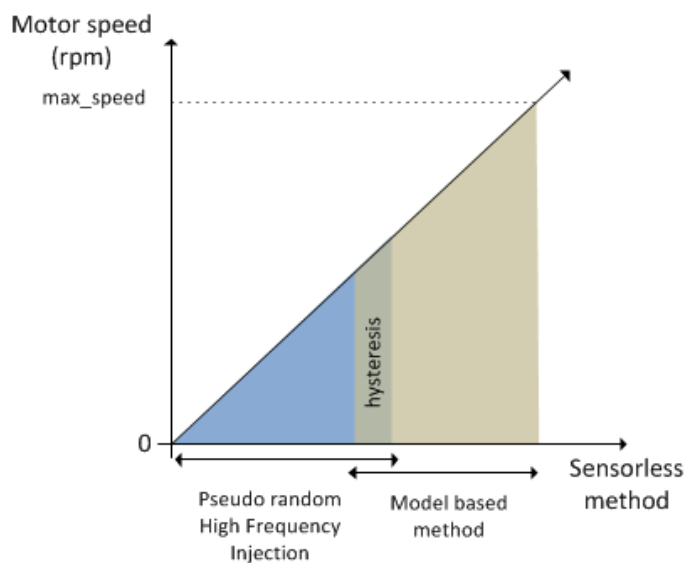

Fig. 3. Transition from High Frequency injection to Back EMF observer

and are modelled in equivalent circuit calculations within the observer. The alpha betacurrents can thus beestimated based on a simplified R/L circuit of the motor. Any deviation between the es timated and actual alpha/beta currents is due to the alpha/beta backEMF voltages. An atan calculation is thus performed to obtain the rotor angle from the es timated back EMF voltages. The subtraction or addition of 90 degrees depending on the rotation direction relates to how the sign of $\omega$ in equation V.2 affects the atan calculation. Note that following the transition to the back EMF method High Frequency Injection is switched off to minimize losses, acoustic noise and optimize the efficiency of the motor.

$\left[\begin{array}{l}V_{\alpha} \\ V_{\beta}\end{array}\right]=R_{s} *\left[\begin{array}{l}i_{\alpha} \\ i_{\beta}\end{array}\right]+L_{s} * \frac{d}{d t}\left[\begin{array}{l}i_{\alpha} \\ i_{\beta}\end{array}\right]+k_{E} \omega *\left[\begin{array}{c}-\sin (\theta) \\ \cos (\vartheta)\end{array}\right]$ Eq V.1

$\left[\begin{array}{l}e_{\alpha} \\ e_{\beta}\end{array}\right]=k_{E} \omega *\left[\begin{array}{c}-\sin (\theta) \\ \cos (\vartheta)\end{array}\right]$

Eq V.2 


\section{Initial position calculation consideration:}

One additional consideration is that the High Frequency Injection method calcu lates the orientation of the rotor but not its polarity. To identify whether $\theta_{e}=\frac{2 \theta_{e}}{2} \operatorname{or} \theta_{e}=\frac{2 \theta_{e}}{2}+\pi$ it is necessary to identify the rotor' $s$ initial position by injecting voltage in the $\mathrm{d}$ axis for each of the two angle assumptions above[19]. The rotor polarity is identified by whichever of the two angle assumptions resulted into higher d axis feedback current. This saturation is only needed to be performed once and is then tracked by the demodulation algorithm.

\section{Simulation}

To evaluate the effectiveness of the proposed sensorless method towards the increase in availability, simulation has been performed to show that High Frequency injection in conjunction with the selected closed loop observer can reliably control the targeted motor. The SPMSM platform targeted for simulation and hardware testing is the Helicopter Electro-Mechanical Actuation System(HEMAS) that is used for the control of the s wash-plate onboard a helicopter. The parameters of the HEMAS motor are lis ted in Table II. Matlab has been used to model sensorless DSP operations that were later implemented in RTL VHDL (targeting FPGAs) and C (targeting DSPs). The RTL VHDL was then simulated incorporating both digital and analogue components including the DUT, the motor model and ADCs.

The simulation covers two scenarios, firstly the HFI is performed at constant frequency (acoustic noise suppression disabled) and secondly where high frequency injection is based on a pseudo random frequency pattern (acoustic noise suppression enabled). The simulation covers both low speed where high frequency injection is used and medium speed where the closed loop back EMF method takes over.

\begin{tabular}{|l|l|}
\hline \multicolumn{1}{|c|}{ Parameter } & \multicolumn{1}{c|}{ Value } \\
\hline No. of pole pairs $\mathrm{p}$ & 5 \\
\hline Rated Current & $4.7[\mathrm{~A}]$ \\
\hline Peak Current & $34[\mathrm{~A}]$ \\
\hline Peak Power & $2.6[\mathrm{~kW}]$ \\
\hline Phase Resistance Rs & $0.23[\Omega]$ \\
\hline Phase Inductance L & $1.3[\mathrm{mH}]$ \\
\hline Voltage constant KE & $0.092[\mathrm{~V} / \mathrm{rads}]$ \\
\hline Torque constant KT & $0.142[\mathrm{Nm} / \mathrm{A}]$ \\
\hline
\end{tabular}

\section{TABLE II. PARAMETERS OF THE HEMAS SPMSM}

\section{A. Pulsating constant HF injection and back EMF method} applied to the HEMAS SPMSM (noise suppression off):

The sensorless block calculates the motor's position at standstill and low speed by applying constant HF sinusoidal signal injection to the estimated synchronous $d$ axis.

$\widehat{V} d_{\text {injected }}=-\operatorname{Vcarrier} *\left(\sin \omega_{c} t\right)$

Eq VII.1

This injection results into stator iron saturation and to the below es timated feedback currents while in saturation

$\widehat{I_{d}}=\frac{\text { Vcarrier } * \cos \left(\omega_{c} t\right)}{\omega_{c} * \mathrm{~L}_{d} * \mathrm{~L}_{q}} *\left(\Sigma \mathrm{L}+\Delta \mathrm{L} \cos \left(2\left(\hat{\theta}_{e}-\theta_{e}\right)\right)\right)$ Eq VII.2

$\widehat{I_{q}}=\frac{\text { Vcarrier } * \cos \left(\omega_{c} t\right)}{\omega_{c} * \mathrm{~L}_{d} \mathrm{~L}_{q}} *\left(-\Delta \operatorname{Lsin}\left(2\left(\hat{\theta}_{e}-\theta_{e}\right)\right)\right) \quad$ Eq VII.3

where $\Sigma \mathrm{L}=\frac{\mathrm{L}_{d}+\mathrm{L}_{q}}{2} \Delta \mathrm{L}=-\frac{\mathrm{L}_{d}-\mathrm{L}_{q}}{2}$ is estimated saliency angle and $\theta_{e}$ is actual saliency angle

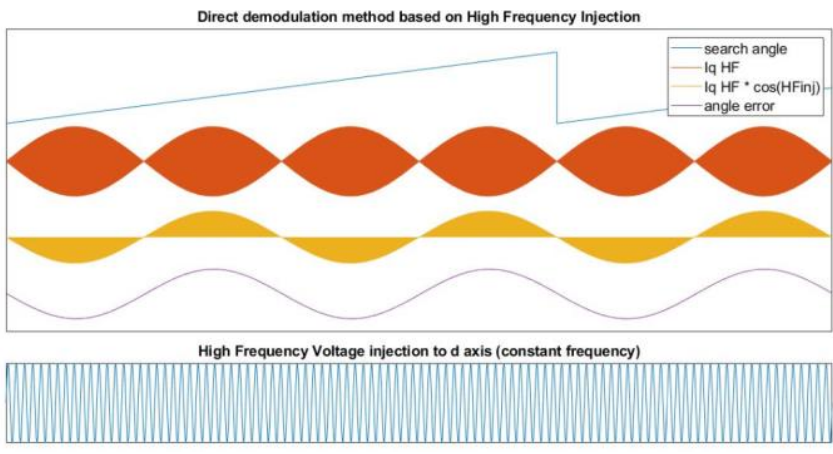

Fig. 4. Matlab simulation, constant HF signal injection in d RRF and direct demodulation

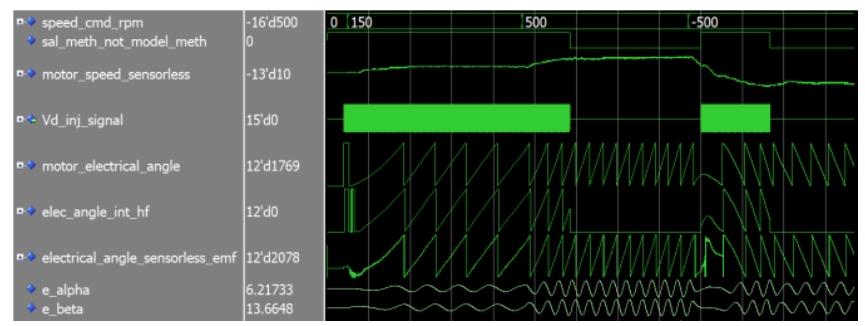

Fig. 5. Modelsim RTL VHDL simulation constant HF signal injection in d $\mathrm{RRF}$ and direct demodulation to back EMF model based transition

By maintaining es timated feedback current $\left(\widehat{I}_{q}\right)$ close to 0 and using this as an error signal the angle error $2\left(\hat{\theta}_{e}-\theta_{e}\right)$ is also maintained to zero. The demodulation method chosen is direct demodulation method and involves applying a low pass filter the aboveresult and use this as an error to align $\hat{\theta}_{e}$ to $\theta_{e}$.

Analysis of results: Matlab simulation was useful towards illustrating the demodulation steps. VHDL simulation shows sensorless control is able to control the motor. At standstill and low speed constant frequency HFI is the active sensorless method and at medium speed HFI is stopped switching toback EMF. When accelerating at opposite direction, there is transition back to HFI at low s peed and then transition to back EMF at medium speed. Also note that the Matlab simulation in Figure 4 assumes constant $\Sigma \mathrm{L}$ and $\Delta \mathrm{L}$ aiming to show clearly the demodulation steps. In practice $\Sigma \mathrm{L}$ and $\Delta \mathrm{L}$ willnot be constants if the angle error decreases substantially as is the case in Matlab simulation. The RTL VHDL simulation however has not made this assumption and was accurate in that respect modelling the dy namic change of Ld/Lq versus Id.

\section{B. Pseudo Random HF injection and back EMF method} applied to the HEMAS SPMSM (noise suppression on):

The sensorless block calculates the motor's position at standstill and slow speed by applying pseudo random HF sinusoidal signal injection to the estimated synchronous $\mathrm{d}$ axis.

$$
V d_{\text {injected }}=-V_{\text {var }} * \sin \left(2 \pi f_{\text {random }} t\right) \quad \text { Eq VII.4 }
$$

where $f_{\text {random_min }} \leq f_{\text {random }} \leq f_{\text {random_max }}$

and $V_{\text {var }}=c * f_{\text {random }}$

Note that the injected voltage needs to vary with frequency to obtain the same amplitude HF feedback current. The demodulation alg orithmis identical to constant frequency $\mathrm{HF}$ sinusoidal injection with the difference that the Band Pass Filter is wider in order to allow for the complete frequency spectrumgenerated by the pseudo randomalgorithm. 


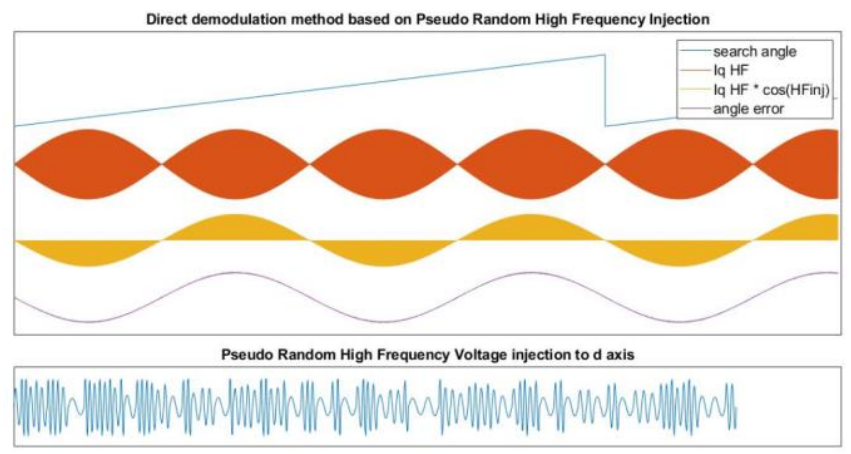

Fig. 6. Matlab simulation, pseudo random sinusoidal high frequency injection in d RRF

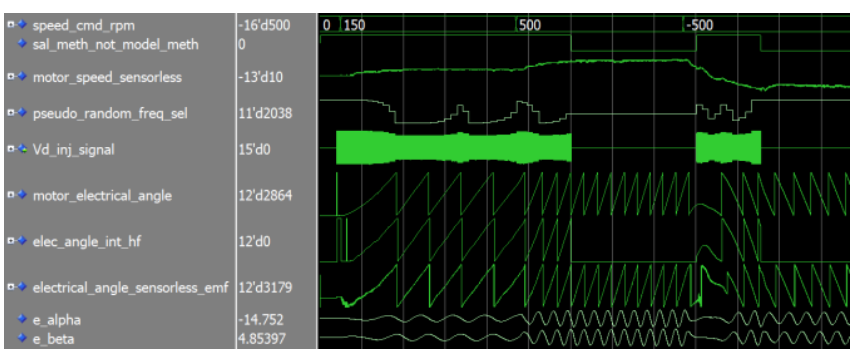

Fig. 7. Modelsim RTL VHDL simulation pseudo random sinusoidal high frequency injection in $\mathrm{R} R \mathrm{RF}$ to back EMF model based transition

Analysis of results: VHDL simulation shows sensorless control is able to control the motor. At standstill and low speed Pseudorandom HFI is the active sensorless method and at medium speed HFI is stopped switching to back EMF. When accelerating at opposite direction, there is transition back to HFI at low speed and then transition to back EMF at medium speed.

\section{CONCLUSIONS}

The possible use of a sensorless method towards an increase in systemavailability was firstanalysed. A survey of sensorless schemes and the proposed hybrid sensorless methodology were then presented. Simulation results in Matlab and at implementation level showed that the proposed hybrid sensorless method was able to control the targeted SPMSM therefore contributing to increase in system availability in case of a res olver failure.

\section{REFERENCES}

[1] R. Dhaouadi, N. Mohan and L. Norum, "Design and implementation of an Extended Kalman filter for state estimation of a permanent magnet synchronous motor', IEEE Trans. Power. Electron., vol. 6, pp. 491497, 1991.

[2] K. Sahri, M. Pietrzak-David, M. Fadel, A. Kheloui, "Sensorless Tolerant Fault Control for Dual Permanent Magnet Synchronous Motor Drive with Global FPGA Emulator", 2018 20th European Conference on Power Electronics and Applications (EPE'18 ECCE Europe), 2018

[3] Muhammed Fazlur Rahman , Sanjeet K. Dwivedi, "Modeling, simulation and control of electrical drives", October 2019, Publisher: Institution of Engineering and Technology

[4] Y. Iwaji, R. Takahata T. Suzuki, M. Hano "All-Speed-Range Drive for Surface Permanent Magnet Synchronous Motors by Combined Use of Two Position Sensorless Methods" 2014 IEEE 5th International Symposium on Sensorless Control for Electrical Drives

[5] Haiyang Tang,Haixia Li ,Jican Lin "Research on Sensorless Control Method of PMSM Based on a Kalman Filter Sliding Mode Observer

[6] S. Medjmadj, D. Diallo, M. Mostefai, C. Delpha, and A. Arias, "PMSM drive position estimation: contribution to the highfrequency injection voltage selection issue," IEEE Trans. Energy Convers., vol. 30, no. 1, pp. 349-358, 2015.
[7] W. Chongwu, H. Yuyao, L. Hong “The study on the PMSM sensorless control using the sub-optimal fading extend Kalman filter", 2013 IEEE 10th International Conference on Power Electronics and Drive Systems (PEDS)

[8] H. Li, Z. Wang "Sensorless Control for PMSM Drives Using the Cubature Kalman Filter based Speed and Flux Observer" 2018 IEEE International Conference on Electrical Systems for Aircraft, Railway, Ship Propulsion and Road Vehicles \& International Transportation Electrification Conference (ESARS-ITEC)

[9] C. Moon, Kee H. Nam, M. K. Jung, C. H. Chae, Y. A. Kwon, "Sensorless speed control of permanent magnet synchronous motor using Unscented Kalman Filter “ 2012 Proceedings of SICE Annual Conference (SICE)

[10] Y. Chen, T. Fu, S. Xing, R. Tang, "Sensorless Control for Permanent Magnet Synchronous Motor Using Sliding Mode Observer" 2006 6th World Congress on Intelligent Control and Automation

[11] A. Accetta, M. Cirrincione, M. Pucci "Sensorless Control of PMSM by a Linear Neural Network: TLS EXIN Neuron" IECON 2010 - 36th Annual Conference on IEEE Industrial Electronics Society

[12] R. Ni, K. Lu, F. Blaabjerg, D. Xu , “A comparative study on pulse sinusoidal high frequency voltage injection and INFORM methods for PMSM position sensorless control" IECON 2016 - 42nd Annual Conference of the IEEE Industrial Electronics Society

[13] J.M. Liu, Z.Q. Zhu "Sensorless control based on third harmonic backEMF and PLL for permanent magnet synchronous machine“, 2013 International Conference on Electrical Machines and Systems (ICEMS)

[14] G. Wang, G. Zhang, D. Xu, "Position Sensorless Control Techniques for Permanent Magnet Synchronous Machine Drives", Springer December 2019

[15] C. Caruana, G. M. Asher and J. Clare, "Sensorless Flux Position Estimation at Low and Zero Frequency by measuring Zero-Sequence Current in Delta Connected Cage Induction Machines", 38th IAS Annual Meeting on Conference Record of the Industry Applications Conference, 2003.

[16] Rodriguez Valiente A, Trinidad A, Garcia Berrocal JR, Gorriz C, Ramirez Camacho R, "Review: Extended high-frequency $(9-20 \mathrm{kHz})$ audiometry reference thresholds in healthy subjects", (April 2014). Int J Audiol. 53 (8): 531-545.

[17] A.Malfait R.Reekmans R.Belmans, "Audible noise and losses in variable speed induction motor drives with IGBT inverters-Influence of the squirrel cage design and the switching frequency", 1994, Proceedings of 1994 IEEE Industry Applications Society Annual Meeting

[18] Hui Jiang Audible noise reduction in the high frequency injection based sensorless torque control for EPS applications, PhD thesis, University of Nottingham, 2012

[19] G. Wang, R. Yang, Y. Wang, Y. Yu, and D. Xu, "Initial Rotor Position Estimation for Sensorless Interior PMSM with Signal Injection", 2010 International Power Electronics Conference, 2010

[20] RTCA/DO-254 Design Assurance Guidance For Airborne Electronic Hardware, RTCA, April 192000

[21] Z. Baozhou, W. Lili, L. Aiying, L. Fuqiang, Y. Chao, D. Jianliang, "Study of a New Detection Technology for Sensorless PMSM Drive with Flux Linkage Observer", 2011 International Conference on Consumer Electronics, Communications and Networks

[22] Y. Jiang, W. Xu, C. Mu, "Improved SOIFO-based rotor flux observer for PMSM sensorless control", IECON 2017 - 43rd Annual Conference of the IEEE Industrial Electronics Society

[23] J. Moreira and T. A. Lipo, "Modeling saturated AC machines including air gap flux harmonic components," IEEE Trans. Ind. Appl., vol. 28, no. 2, pp. 343-349, Mar./Apr. 1992

[24] C. Varghese, V Suresh, "Control of Permanent Magnet Synchronous Motor using MRAS", International Journal of Latest Trends in Engineering and Technology, Vol. 3, Issue 4, pp. 71-77, March 2014. 